\title{
Perspectives on Advancements in Dental Sleep Medicine
}

Disclaimer: The use, mention or depiction of any product, device, service or appliance shall not be interpreted as an endorsement, recommendation or preference by the AADSM. Any opinion expressed is solely the opinion of the individual, and not that of the AADSM.

The field of dental sleep medicine (DSM) has changed a great deal over the past decade. With new technologies and processes constantly emerging, the field is poised to continue to grow and develop over the next decade as well. We reached out to our members to get their perspective on what exciting advancements the future may hold for dental sleep medicine.

\section{WHAT NEW INNOVATION OR PROCESS DO YOU THINK MAY HAVE THE GREATEST IMPACT ON DSM IN THE FUTURE?}

"The two things I think could really help DSM in the future are:

1. Better technology regarding HSAT. Insurances are making it very hard to have PSG's and patients do not want to pay for them out of pocket. Devices should get smaller. Additionally, a better reading of sleep stages could give us more accurate data.

2. Pharmacology! Medications that could help muscle dilation could yield a more predictable result with severe patients in MAD therapy."

\section{-Dr. Girish Bharwani, DDS, D.ABDSM}

"Of all the exciting developments in the everchanging world of dental sleep medicine, there are two that I find particularly noteworthy. The first is the continuing removal of barriers to the sleep testing process and the increasing participation of dentists in this area. The development of simpler, more user-friendly HST devices--and particularly the ability of dentists (in many states) to use these devices to administer tests to be interpreted by a sleep physician---means that more of the $80-90 \%$ of patients whose OSA is undiagnosed can be identified and treated.

Another factor that I believe has the potential to greatly impact DSM is the evolution of therapies for children to treat childhood SDB. Modalities such as removable oral appliances and myofunctional therapy have the potential to guide children's growth to enable them to develop healthy airways, possibly preventing them from becoming sleep apneic adults.

I am excited about the possibilities raised by both of these developments in which dentists can play key roles in the diagnosis, treatment and perhaps even the prevention of OSA."

-Dr. Joel Nathanson, DMD, D.ABDSM

"We are now living in a precision digital accelerated world. Technology allows us to instantly scan and transmit intraoral images and 3D models. I believe that the transition of DSM to a fully digital workflow will create new opportunities for designers, as well as dental practitioners. I look to see a new class of oral appliances for treatment of OSA, which might include traditional mandibular advancement devices as well as functional orthopedic appliances for reshaping the airway and oral anatomy. In addition to adding new appliances, I see a trend toward in-office fabrication using high speed 3D printers using biocompatible materials. As DSM continues to make its mark in effective treatments for OSA, I believe that research and software groups will be positioned to add these elements to our current offering of services to patients.

I also see that quality education for DSM providers will be needed more than ever. My suggestion for dentists is to find quality partners who understand the quickly evolving landscape of new manufacturing technologies and software. The future is bright for those who see past the limitations of today's tools and techniques!"

\section{-Dr. Paul Jacobs, DDS, D.ABDSM}

"Nowadays, dentists have many tools to address OSA; physical examination, oximetry, PSG, HSAT, OA options, therapies combination, etc.

Although OAT has a high success grade, it's a little bit difficult to predict outcome in all cases. We gather a lot of data to compound our therapy planning. However, 
patients may have several phenotypes those are not easy to discover; Pcrit, arousal threshold, loop gain and muscle responsiveness play an important role in whether the patient has a good or bad outcome.

Many papers already show that soon we can plan our treatments using these data.

But we need to first discover these patients. PSG is, in most countries, expensive, not widely available and even insufficient. According to publication in Lancet in 2019*, the USA has 54 million apneics. In Brazil, my country, there are 49 million. The more delayed the diagnosis, the more impact OSA causes. So, pulse oximetry (with results interpreted by sleep physician) is a great tool. This test must be offered by general dentists and physician. Relying on the currently qualified or diplomate dentists and physician aren't sufficient to find the sufferers; we need more people with a "trained vision".

*Estimation of the global prevalence and burden of obstructive sleep apnea: a literature-based analysis http://dx.doi.org/10.1016/S2213-2600(19)30198-5"

\section{- Dr. Walter Silva Jr., DDS, ABDSM International Certificant}

"There are two methods of Electromyography (EMG): surface EMG which is used in our profession and intramuscular EMG recording.

Usually, surface EMG is used to assess muscle function by recording muscle activity from the surface over the muscle on the skin, using a pair of electrodes. Surface EMG permits a non-invasive investigation of the bioelectrical phenomena of muscle activities of the stomatognatic system. In fact, it's the only way to assess objectively muscle activity (hyper or hypo activity), muscle balance and harmony of Temporalis, Masseters,
Suprahyoid, SCM muscles. As dentists, we do affect those muscles with our treatments on daily basis (orthodontics, orthognatic surgeries, prosthodontics etc). We also may affect those muscles with our oral appliance therapies(OAT) for OSA.

As a practitioner for many years in DSM, I would love to know if the baseline muscle physiology of my patient is pathological or harmonious when a patient is referred to me for OAT. I would love to know if I am respecting the muscle physiology during the bite registration for a starting position of OAT. Also, I would love to know in every mm of titration during OAT, how these muscles are reacting and be able to note that in the patient's chart. Finally, IF and WHEN my patient comes back after using the OAT with side effects like discomfort or muscle pain, surface EMGs will help to manage those side effects.

How do we assess all these without EMGs? Well, we rely on patients' subjective symptoms and the clinical examination with some muscle palpations (which are also subjective).

The value of surface EMG is best expressed by C.J. DeLuca, Professor of Biomedical Engineering and Research and Professor of Neurology at Boston University who said, "Surface EMG utilizes sensing electrodes placed on the skin, which allows the clinician to directly and accurately monitor muscle activity. This is far more accurate procedure than conventional manual palpation or touch which can provide only gross assessments of muscle activity."

In my personal opinion, this technology takes dental sleep medicine to the next level because objective measurement helps us understand more information for an improved patient outcome.

- Dr. Sahag Mahseredjian, DMD, D.ABDSM

\section{CITATION}

Perspectives on advancements in dental sleep medicine. J Dent Sleep Med. 2021;8(3). 

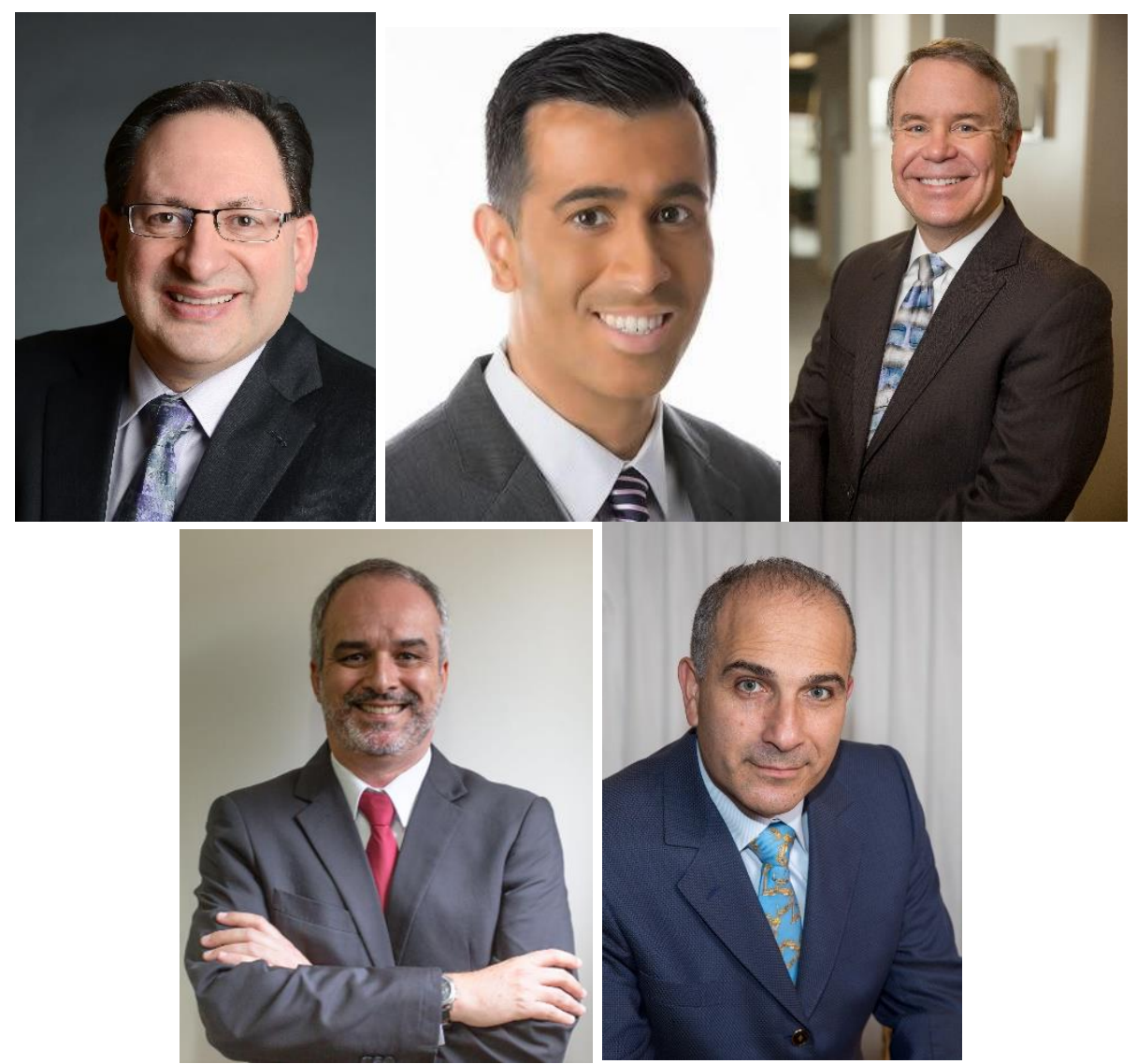

Top Row (Left to Right): Dr. Paul Jacobs, Dr. Girish Bharwani, Dr. Joel Nathanson

Bottom Row (Left to Right): Dr. Walter Silva Jr., Dr. Sahag Mahseredjian 